\title{
Mood and Memory Deficits in a Model of Gulf War Illness Are Linked with Reduced Neurogenesis, Partial Neuron Loss, and Mild Inflammation in the Hippocampus
}

\author{
Vipan K Parihar ${ }^{1,2,6}$, Bharathi Hattiangady ${ }^{1,2,3,4,5,6}$, Bing Shuai ${ }^{1,2,3,4,5}$ and Ashok K Shetty*, 1,2,3,4,5,6 \\ 'Research Service, Durham Veterans Affairs Medical Center, Durham, NC, USA; ${ }^{2}$ Department of Surgery (Neurosurgery), Duke University \\ Medical Center, Durham, NC, USA; ${ }^{3}$ Institute for Regenerative Medicine, Texas A\&M Health Science Center College of Medicine at Scott \& White, \\ Temple, TX, USA; ${ }^{4}$ Department of Molecular and Cellular Medicine, Texas A\&M Health Science Center College of Medicine, College Station, TX, \\ USA; ${ }^{5}$ Research Service, Olin E. Teague Veterans Affairs Medical Center, Central Texas Veterans Health Care System, Temple, TX, USA
}

Impairments in mood and cognitive function are the key brain abnormalities observed in Gulf war illness (GWI), a chronic multisymptom health problem afflicting 25\% of veterans who served in the Persian Gulf War-I. Although the precise cause of GWI is still unknown, combined exposure to a nerve gas prophylaxis drug pyridostigmine bromide (PB) and pesticides DEET and permethrin during the war has been proposed as one of the foremost causes of GWI. We investigated the effect of 4 weeks of exposure to Gulf war illness-related (GWIR) chemicals in the absence or presence of mild stress on mood and cognitive function, dentate gyrus neurogenesis, and neurons, microglia, and astrocytes in the hippocampus. Combined exposure to low doses of GWIR chemicals PB, DEET, and permethrin induced depressive- and anxiety-like behavior and spatial learning and memory dysfunction. Application of mild stress in the period of exposure to chemicals exacerbated the extent of mood and cognitive dysfunction. Furthermore, these behavioral impairments were associated with reduced hippocampal volume and multiple cellular alterations such as chronic reductions in neural stem cell activity and neurogenesis, partial loss of principal neurons, and mild inflammation comprising sporadic occurrence of activated microglia and significant hypertrophy of astrocytes. The results show the first evidence of an association between mood and cognitive dysfunction and hippocampal pathology epitomized by decreased neurogenesis, partial loss of principal neurons, and mild inflammation in a model of GWI. Hence, treatment strategies that are efficacious for enhancing neurogenesis and suppressing inflammation may be helpful for alleviation of mood and cognitive dysfunction observed in GWI.

Neuropsychopharmacology (20I3) 38, 2348-2362; doi: I0.1038/npp.20 I3. I58; published online 3I July 2013

Keywords: adult neurogenesis; anxiety; depression; learning and memory; mood; neuroinflammation

\section{INTRODUCTION}

Nearly $25 \%$ of 700000 veterans who served in the Persian Gulf War-1 (PGW-1) are diagnosed with Gulf war illness (GWI) (Golomb, 2008; Binns et al, 2008). GWI is a chronic multisymptom health problem that is poorly understood because the underlying pathologies causing different symptoms are unclear. Among the health problems, illnesses affecting the brain and musculoskeletal system appear to be more prevalent (Amato et al, 1997; Haley et al, 2000a,b; Steele, 2000). Brain dysfunction is typified mainly by depression, anxiety, and learning and memory impairments (Haley et al, 2000a,b; Odegard et al, 2012).

*Correspondence: Dr AK Shetty, Institute for Regenerative Medicine, Texas A\&M Health Science Center College of Medicine at Scott \& White, 570I Airport Road, Module C, Temple, TX 7650I, USA, Tel: + 254 77| 6804, Fax: + $25477 \mid$ 6839,

E-mail: Shetty@medicine.tamhsc.edu

${ }^{6}$ These authors contributed equally to this work.

Received 18 February 2013; revised 29 May 2013; accepted 21 June 2013; accepted article preview online 28 June 2013
Although the precise etiology of GWI is unknown, several suspected causes have been proposed. Among these, the hypothesis that GWI is linked to a combination of exposures encountered by service personnel during the PGW-1 has received much attention. First, veterans who were stationed in the battlefield areas were believed to have consumed pyridostigmine bromide (PB) pills daily during the war (Binns et al, 2008). PB was employed as a prophylactic treatment (ie, as a reversible acetylcholinesterase (AChE) inhibitor) to protect against the possible attack with irreversible AChE inhibitors such as organophosphate nerve gas agents (Binns et al, 2008). Second, preparations for the PGW-1 comprised measures to offset infectious diseases transmitted by insects and ticks in the region. The measures included the use of pesticides for the area protection and the use of insect repellants on the skin and the uniform. The pesticides included the insecticide permethrin (PM) and the insect repellant $N, N$-diethyl-mtoluamide (DEET) (Haley and Kurt, 1997). Permethrin, a synthetic pyrethroid, is a widely used insecticide (Vijverberg and van den Bercken, 1990). Permethrin 
intoxication results as a consequence of the sustained opening of voltage-gated sodium channels leading to repetitive discharges after a single stimulus (Narahashi, 1985). The chemical DEET is the most commonly used insect repellant (Veltri et al, 1994). Although the precise target of DEET is unknown, extensive and repeated topical application of DEET leads to human poisoning including death (Chaney et al, 2000).

Thus, in view of the exposure of service personnel to both GWI-related (GWIR) chemicals (PB, PM, and DEET) and war-related stress, it is hypothesized that the neurological symptoms displayed by a significant number of PGW-1 veterans are because of synergistic interaction of $\mathrm{PB}$ with pesticides PM and DEET and/or stress (Binns et al, 2008; Friedman et al, 1996; Hyams et al, 1996; Everson et al, 1999; Steele et al, 2012; Abdullah et al, 2011; Torres-Altoro et al, 2011). This chemical exposure hypothesis is also supported by the report of the Research advisory committee (RAC) on GWI that the overall prevalence of GWI is greater in veterans who used higher amounts of pesticides than veterans who had limited exposure to pesticides during the PGW-1 (Binns et al, 2008). In this study, using a rat model, we investigated whether exposure to GWIR chemicals for 4 weeks would be sufficient to cause anxiety- and depressivelike behavior and/or cognitive dysfunction. Because changes such as reduced neurogenesis, neurodegeneration, or inflammation in the hippocampus may underlie impairments in mood and cognitive function (Bilbo et al, 2012; Deng et al, 2010; Eisch and Petrik, 2012), we also examined whether exposure to GWRI chemicals is associated with declined neurogenesis, loss of principal neurons, activation of microglia, and hypertrophy of astrocytes in the hippocampus.

\section{MATERIALS AND METHODS}

\section{Animals}

Three-month-old Sprague-Dawley rats obtained from Harlan (Indianapolis, IN) were randomly assigned to one of the four major groups ( $n=6 /$ group/time point, total, 48$)$ : (1) vehicle (VEH) group receiving vehicle and handling daily for 4 weeks; (2) chemicals (CHEM) group receiving $\mathrm{PM}$, DEET, and PB, and handling daily for 4 weeks; (3) chemicals plus stress (CHEM + STRESS) group receiving $\mathrm{PM}, \mathrm{DEET}, \mathrm{PB}$, and $5 \mathrm{~min}$ of restraint stress daily for 4 weeks; and (4) naive control group.

\section{Application of Chemicals and Stress}

The chemical PB $(1.3 \mathrm{mg} / \mathrm{kg})$ was administered through oral gavage $(500 \mu \mathrm{l}$ in sterile water). Solutions of DEET (200 $\mu \mathrm{l}$ containing $40 \mathrm{mg} / \mathrm{kg}$ in $70 \%$ alcohol) and PM $(200 \mu \mathrm{l}$, $0.13 \mathrm{mg} / \mathrm{kg}$ in $70 \%$ alcohol) were applied sequentially to fur-clipped skin areas located on the back of neck and the upper thoracic region. A rat restrainer was used for the induction of $5 \mathrm{~min}$ of restraint stress, as described in our previous study (Parihar et al, 2011). The doses of PB, PM, and DEET were chosen based on a previous study (Abdel-Rahman et al, 2002).

\section{Behavioral Analyses}

During the third month after the exposure, animals in all groups ( $n=6$ /group) were tested for mood and cognitive function using a variety of behavioral analyses in the following sequence: elevated plus maze test (EPMT, for assessing anxiety-like behavior), novel object recognition test (NORT, for examining object recognition memory), water maze test (WMT, for investigating spatial learning and memory), and forced swim test (FST, for assessing depressive-like behavior).

\section{Analyses of Anxiety- and Depressive-Like Behavior}

Animals were examined with EPMT and FST, as described in our recent reports (Parihar et al, 2011; Hattiangady and Shetty, 2012) and the Supplementary File. Numbers of open arm entries and times spent in open arms were considered as indices of anxiety-like behavior, and the amount of time spent in floating in FST was used as an index of depressivelike behavior.

\section{Measurement of Recognition Memory Function}

Recognition memory was measured using NORT, as described elsewhere (Broadbent et al, 2009; Parihar et al, 2011) and the Supplementary File.

\section{Assessment of Spatial Learning and Memory Function}

Spatial learning and memory function was measured through a WMT, as detailed in our recent reports (Parihar et al, 2011; Hattiangady and Shetty, 2012) and the Supplementary File.

\section{Analyses of Hippocampal Neurogenesis}

For analyses at an early time point, subgroups of animals from every group ( $n=6 /$ group) received intraperitoneal injections of $5^{\prime}$-bromodeoxyuridine (BrdU; $50 \mathrm{mg} / \mathrm{kg}$, once every $6 \mathrm{~h}$ for $18 \mathrm{~h}$ ) immediately after the exposure regimen. Animals were perfused $6 \mathrm{~h}$ after the last BrdU injection and brain tissues processed for BrdU immunohistochemistry, BrdU and doublecortin (DCX) dual immunofluorescence and confocal microscopy, and DCX immunohistochemistry. For analyses of neurogenesis at an extended time point, animals that underwent behavioral tests were used. A week after the completion of behavioral tests (ie, in the fourth month after the exposure), animals received intraperitoneal injections of $\mathrm{BrdU}$ ( $100 \mathrm{mg} / \mathrm{kg}$; once daily for 12 days). Animals were perfused a month after the last BrdU injection and brain tissues were processed for BrdU immunohistochemistry, BrdU and neuron-specific nuclear antigen (NeuN) dual immunofluorescence and confocal microscopy, and DCX immunohistochemistry ( $n=4-6$ animals/ group), as detailed in our previous reports (Hattiangady and Shetty, 2010; Parihar et al, 2011).

\section{Measurement of Numbers of Newly Born Cells and Neurons}

The numbers of newly born $(\mathrm{BrdU}+)$ cells and newly born $(\mathrm{DCX}+)$ neurons in the subgranular zone-granule cell layer 
(SGZ-GCL) of the dentate gyrus (DG) were measured from $30-\mu \mathrm{m}$-thick serial sections (every 15th) through the entire hippocampus via an optical fractionator method using StereoInvestigator, as detailed in the Supplementary File.

\section{Quantification of Neuronal Differentiation of Newly Born Cells and Net Neurogenesis}

We quantified the percentages of BrdU + newly born cells in the SGZ-GCL expressing: (1) DCX in tissues collected $6 \mathrm{~h}$ after the last of four BrdU injections; and (2) NeuN in tissues collected 1 month after the last of 12 daily BrdU injections, using methods described in our earlier report (Hattiangady and Shetty, 2010). We measured net neurogenesis using data such as total numbers of BrdU + cells and percentages of newly born cells that differentiate into $\mathrm{DCX}+$ or NeuN + neurons.

\section{Analyses of NSCs in the Hippocampus}

Serial sections (every 15th) through the entire hippocampus from tissues harvested for long-term neurogenesis analyses were processed for visualization of Sox-2 (a marker of neural stem cell (NSCs) and a subpopulation of astrocytes; Hattiangady and Shetty, 2008). The number of Sox- $2+$ cells in the SGZ was quantified ( $n=4$ animals/group) as described in our earlier report (Hattiangady and Shetty, 2008) and the Supplementary File. Next, we performed dual immunofluorescence for Sox-2 and S-100 $\beta$ and quantified fractions of Sox- $2+$ cells expressing S-100 $\beta$ (mature astrocytes) and Sox- $2+$ cells lacking S-100 $\beta$ (putative NSCs) using a confocal microscope. We estimated the numbers of putative NSCs in the SGZ using data such as total numbers of Sox- $2+$ cells and percentages of Sox- $2+$ cells that lacked S-100 $\beta$. To ascertain the proliferative behavior of NSCs, we performed dual immunofluorescence for Sox-2 and Ki-67 (an endogenous marker of proliferating cells) and quantified fractions of Sox- + cells expressing Ki-67. We appraised the numbers of putative NSCs in the SGZ exhibiting proliferation through data such as total numbers of Sox- $2+$ cells and percentages of Sox- $2+$ cells that express Ki-67.

\section{Analyses of Hippocampal Neurodegeneration, Volume, and Inflammation}

Serial sections (every 15th) through the entire hippocampus from tissues harvested for long-term neurogenesis analyses above were processed for NeuN immunostaining (Shetty et al, 2009). Next, the numbers of $\mathrm{NeuN}+$ neurons in different cell layers of the hippocampus were stereologically measured ( $n=5$ /group; Shetty et al, 2009 and Supplementary File). Volumes of DG, CA1 and CA3 subfields, and the entire hippocampus (including fimbria) were measured through tracing of hippocampal regions and the entire hippocampus (including fimbria) in serial sections ( $n=5$ animals/group) using Neurolucida (Microbrightfield). Additional sets of serial sections (every 20th) were processed for visualization of all astrocytes (GFAP immunohistochemistry), reactive astrocytes (nestin and vimentin immunohistochemistry), and activated microglia (ED-1 immunohistochemistry) using methods described elsewhere (Abdel-Rahman et al, 2004b; Hattiangady et al, 2004). The numbers of ED-1 + cells were quantified per section, whereas GFAP + structures in different regions of the hippocampus were quantified through area fraction analysis using the software program ImageJ, as described in our earlier report (Shetty et al, 2004) and Supplementary Data File.

\section{Characterization of the Effects of GWIR Chemicals on NSC Proliferation In Vitro}

Hippocampi of postnatal day -1 rat pups were dissected, triturated, and processed as single-cell suspension (Hattiangady and Shetty, 2012). One hundred live cells suspended in $0.5 \mathrm{ml}$ of NSC proliferation medium were plated per culture well in a 24-well plate in the presence of individual or combinations of GWIR chemicals (each at a concentration of $100 \mu \mathrm{M} ; n=16$ culture wells/group). Cells were allowed to proliferate for 7 days. The numbers of neurospheres/100 live cells plated in different treated groups were measured to ascertain the extent of NSC proliferation.

\section{Data Analyses}

Values (mean \pm SEM) from different groups were compared using one-way ANOVA followed by Newman-Keuls multiple comparison post-tests. For learning analyses in WM tests, additional tests such as one-way and two-way repeated measures ANOVA (RM-ANOVA) with Bonferroni multiple comparisons post hoc tests were employed. Because VEH-treated rats did not differ from naive controls for any of the parameters measured, the experimental groups were compared with the VEH-treated group in all analyses.

\section{RESULTS}

\section{Exposure to GWIR Chemicals Did Not Alter Body Weights or General Behavior of Animals}

Exposure to CHEM or CHEM + STRESS did not cause major health defects in animals. This was evidenced by comparable increases in body weights between VEH-treated animals and animals treated with CHEM or CHEM + STRESS in the exposure period as well as 2 months after the exposure $(p>0.05, \mathrm{~F}=0.96$, two-way ANOVA; Supplementary Figure S1 and Supplementary Data File). Moreover, animals did not display discoloration of the fur or any other signs of significant pain and discomfort (such as porphyrin around eyes/nose, poor grooming, decreased mobility, or head tucked hunched posture).

\section{Exposure to GWIR Chemicals Induced Depressive- and Anxiety-Like Behavior}

Animals treated with CHEM or CHEM + STRESS exhibited increased levels of depressive- and anxiety-like behavior in FST and EPMT in comparison with VEH-treated animals (Figure 1). This was revealed through greater amount of time spent in immobility (or floating) in FST $(p<0.0001$, $\mathrm{F}=20.7$; Figure 1a2), and fewer entries into open arms $(p<0.0001, \mathrm{~F}=28.1$; Figure 1b1) and reduced dwell time in 
open arms in EPMT $(p<0.0001, \mathrm{~F}=22.9$; Figure 1b2). Furthermore, animals treated with CHEM + STRESS exhibited greater immobility time and fewer open arm entries than animals treated with CHEM alone $(p<0.05-0.01$, Figure $1 \mathrm{a} 2$ and $\mathrm{b} 1$ ).

\section{Exposure to GWIR Chemicals Resulted in Spatial Learning and Memory Dysfunction}

Analyses of swim speeds revealed no statistical differences among the three groups. However, animals exposed to CHEM + STRESS exhibited $17 \%$ reduced swim speed than VEH-treated rats (Figure 1a]). Therefore, all learning data were analyzed using both swim path lengths and latency values. Both parameters revealed comparable findings and hence only swim path length data are illustrated (Figure 2a2). One-way RM-ANOVA on swim path lengths to reach the hidden platform over the learning sessions revealed that animals belonging to all three groups have the ability for spatial learning $(p<0.0001$; F-values: $\mathrm{VEH}=39.9, \quad \mathrm{CHEM}=7.5, \quad$ and $\mathrm{CHEM}+\mathrm{STRESS}=11.1$;
Figure 2a2). However, two-way RM-ANOVA revealed that exposure to GWIR chemicals had significant effect on learning curves $(p<0.01, \mathrm{~F}=8.5)$. The post hoc tests revealed that swim path lengths were significantly longer in CHEM and CHEM + STRESS groups than the VEH group in the third learning session $(p<0.05-0.01$; Figure 2a2). Furthermore, analyses of swim path efficiency (the ratio of the shortest possible swim path length to actual swim path length) using two-way RM-ANOVA demonstrated that exposure to GWIR chemicals affected learning $(p<0.0001$, $\mathrm{F}=23.03$ ). Animals exposed to CHEM or CHEM + STRESS displayed swim path efficiencies in the last five sessions of learning that were inferior to VEH-treated rats $(p<0.05-$ 0.0001; Figure 2a3). We also measured activity in the thigmotactic zone (TZ), an annular area measuring $18 \mathrm{~cm}$ width along the periphery of the pool. Activity in the TZ refers to an animal's propensity to move along the edge of its environment, and swimming for prolonged periods in the TZ during the course of WMT is attributed to anxiety or fear (Treit and Fundytus, 1998; Herrero et al, 2006). However, animals exposed to CHEM or CHEM + STRESS a1
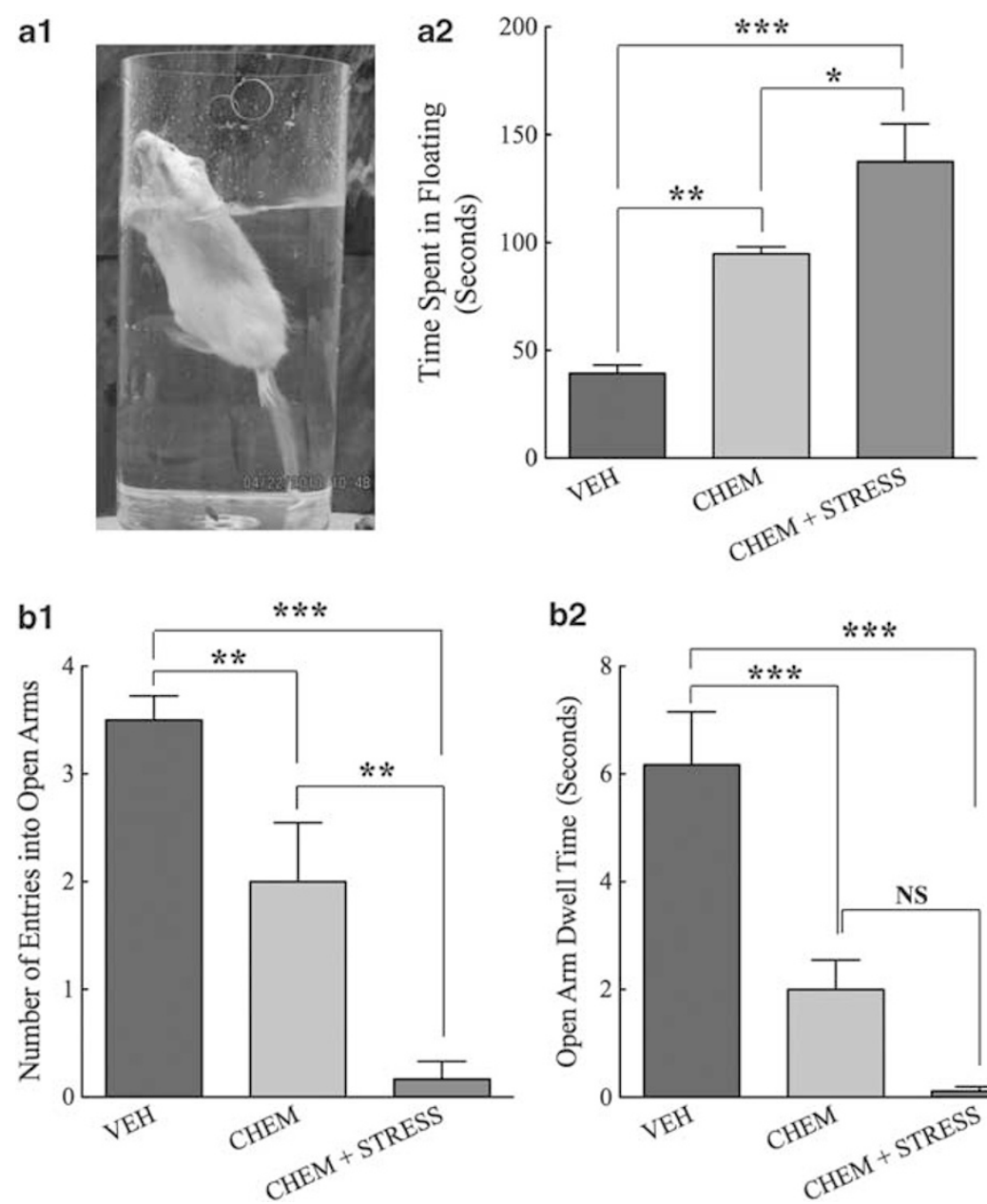

b2

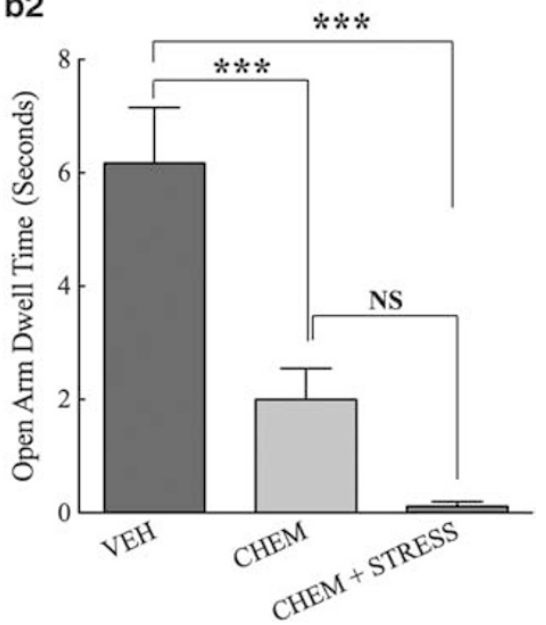

Figure I Exposure of adult rats to Gulf war illness-related chemicals results in increased depressive- and anxiety-like behavior, and addition of mild stress during the period of exposure exacerbates the detrimental effects of chemicals on mood function. (al) A rat undergoing forced swim test (FST) is shown. (a2) The bar chart demonstrates that rats exposed to chemicals (CHEM group) and chemicals and stress (CHEM+ STRESS group) spend greater amount of time in floating (a measure of depressive-like behavior) in FST in comparison with vehicle-treated control rats (VEH group). (bl, b2) Bar charts show that exposure of rats to CHEM or CHEM + STRESS results in considerably fewer entries into open arms and reduced open arm dwell times (indices of anxietylike behavior) in an elevated plus maze (EPM) test in comparison with rats exposed to VEH. ${ }^{*} p<0.05$; $* *$ * $p<0$ l; **** $p<0.00$ I. NS, not significant. 
did not display increased anxiety in the WMT, which was evidenced through comparable swim path lengths in the TZ between rats belonging to these two groups and the VEH group (Figure 2a4, two-way RM-ANOVA, $p>0.05, \mathrm{~F}=1.7$ ). In addition, comparable average latency values to reach the platform in the visible platform test revealed similar visual
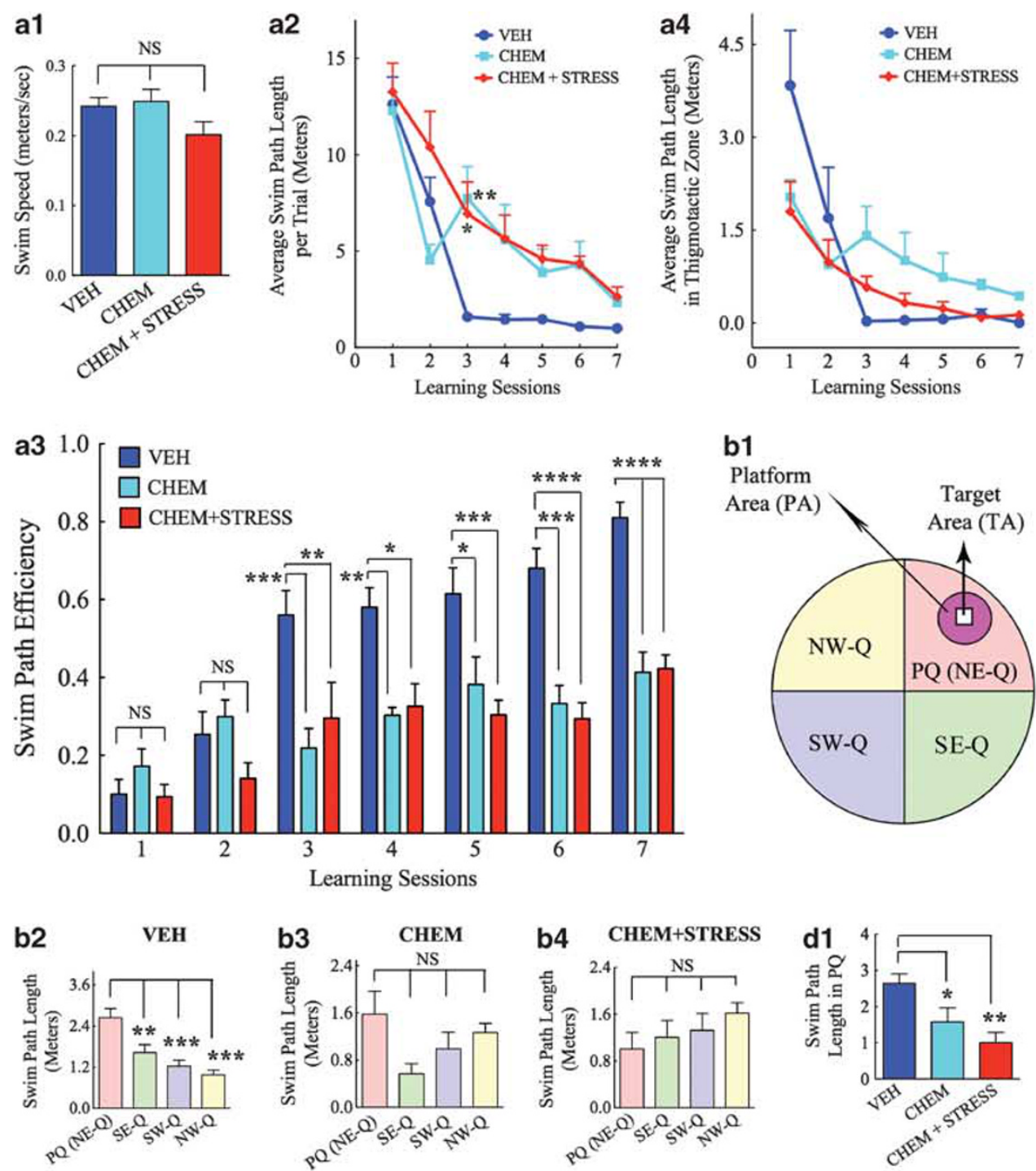

b1

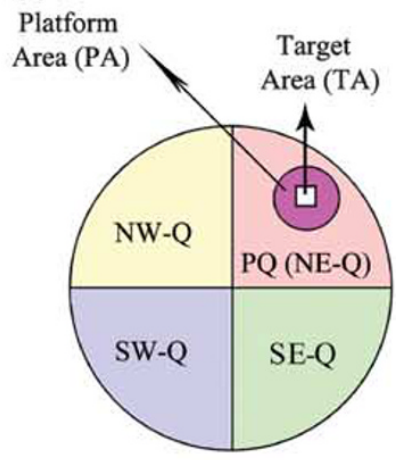

Learning Sessions
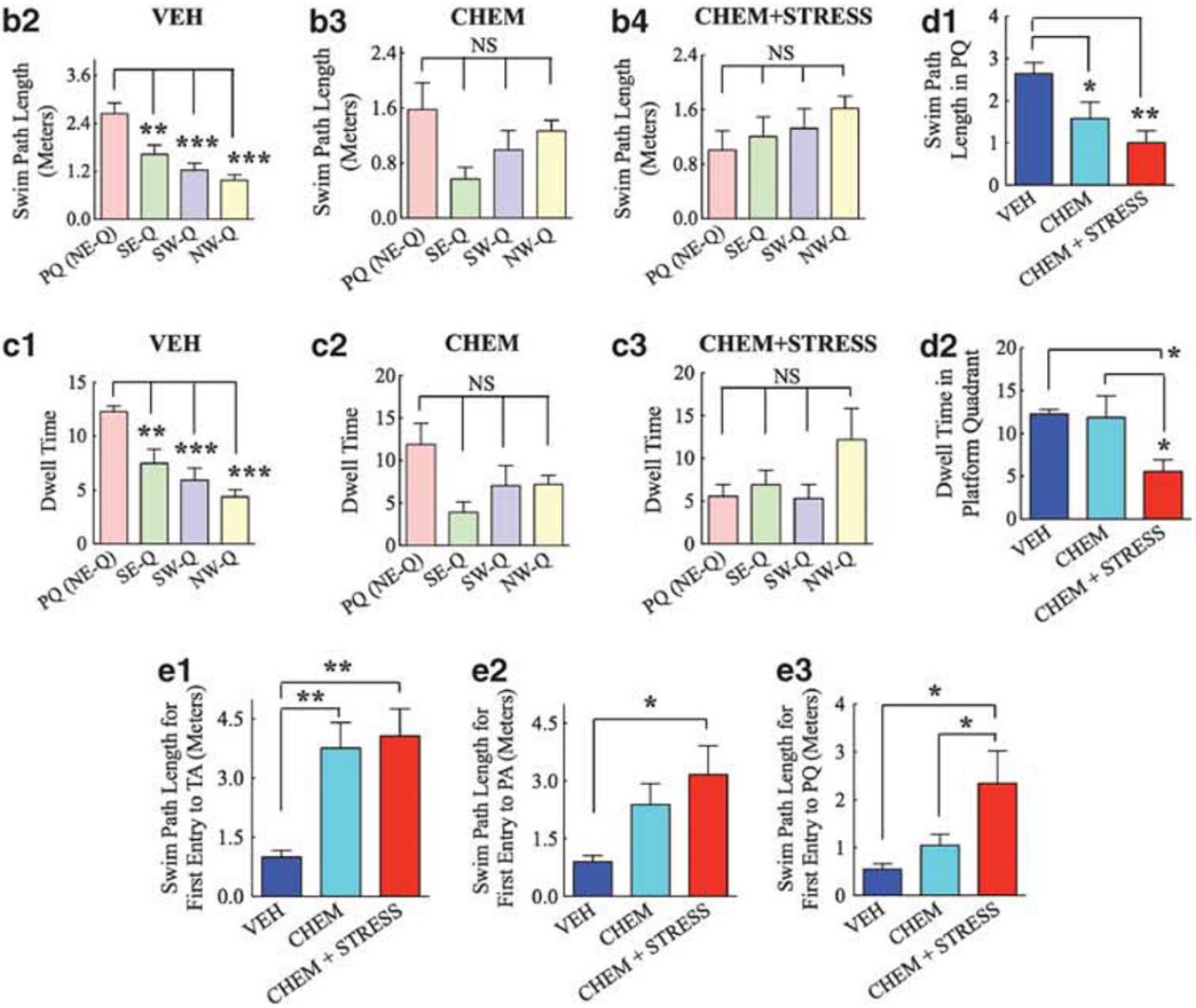
sensory function in the three groups of rats (mean \pm SEM in seconds: VEH, $7.5 \pm 2.4$; CHEM, $5.9 \pm 0.9$; and CHEM + STRESS, $7.8 \pm 1.6, p>0.05, \mathrm{~F}=0.35)$. Thus, learning deficits seen in animals exposed to CHEM or CHEM + STRESS are not related to increased anxiety, motor disability, or visual sensory dysfunction. Data pertaining to activity in the $\mathrm{TZ}$ further implied that animals in these groups were able to focus on the learning task but their overall ability for learning was inferior to VEH-treated rats.

Rats treated with VEH spent greater amount of the probe test time in the platform quadrant $(\mathrm{PQ}$, a quadrant of the pool where platform was placed during learning sessions) in comparison with all other quadrants. This was evidenced through ANOVA analyses of swim path lengths and dwell times in different quadrants $(p<0.0001, \mathrm{~F}=12.6$; Figure $2 \mathrm{~b} 2$ and $\mathrm{cl}$ ). In contrast, rats exposed to CHEM or CHEM + STRESS did not display greater affinity for the PQ vis-à-vis the other three quadrants $(p>0.05, F=0.9-2.9$, Figure $2 b 3$, b4, c2, andc3). Memory retrieval dysfunction in rats exposed to CHEM or CHEM + STRESS was also apparent from shorter swim path lengths in the PQ than rats in the VEH group $(p<0.05-0.01$, Figure $2 \mathrm{~d} 1)$. Rats exposed to CHEM + STRESS also exhibited reduced dwell time in the PQ than VEH- or CHEM-treated rats $(p<0.05$, Figure $2 \mathrm{~d} 2)$. Furthermore, rats exposed to CHEM or CHEM + STRESS displayed longer swim path lengths for first entry to target area (TA, an area denoting the actual position of the platform, $p<0.01$, Figure $3 \mathrm{e} 1)$. Rats exposed to CHEM + STRESS in addition showed longer swim path length values for their first entry to platform area (PA, an annular area denoting the position of the platform as well as the immediate surrounding region) and PQ than rats treated with VEH $(p<0.05$, Figure $2 \mathrm{e} 2$ and e3).

As rats exposed to CHEM + STRESS exhibited greater level of depressive- and anxiety-like behavior in FST and EPMT than other groups, we examined whether greatly impaired memory function in these rats are linked to increased despair through a principal component analysis (PCA). This analysis showed that memory dysfunction contributed to $40 \%$, depression to $35 \%$, and anxiety to $24 \%$ of the variance in principal component 1 . Thus, analyses of principal components and thigmotactic activity in WMT together suggested that memory dysfunction, depression, and anxiety are comorbidities in this prototype of GWI. The results of PCA are detailed in the Supplementary Data File.

\section{Exposure to GWIR Chemicals Did Not Impair Recognition Memory Function}

Rats exposed to CHEM + STRESS spent greatly reduced time in object exploration, in comparison with both VEHand CHEM-treated rats $(p<0.0001, \mathrm{~F}=18.1$; Supplementary Figure S2a1). Hence, an average time spent with the novel object by these rats was much less than that observed for VEH- or CHEM-treated rats $(p<0.001, F=12.4$; Supplementary Figure S2a2). However, the percentage of the total object exploration time spent with the novel object (object discrimination index) was comparable across the three groups $(p>0.05, \mathrm{~F}=0.9$; Supplementary Figure S2a3).

\section{Exposure to GWIR Chemicals Resulted in Reduced Hippocampal Neurogenesis}

In comparison with VEH-treated animals, animals exposed to CHEM or CHEM + STRESS displayed reduced production of new cells per day $(p<0.0001, F=70.1$; Figure $3 a 1$ to $d)$, no changes in neuronal differentiation of newly born cells $(p>0.05, \mathrm{~F}=3.3$; Figure $3 \mathrm{e} 1$ and $\mathrm{f})$, diminished net neurogenesis $(p<0.0001, \mathrm{~F}=98.4$; Figure $3 \mathrm{~g})$, and reduced levels of ongoing neurogenesis (based on numbers of DCX + immature neurons, $p<0.0001, \mathrm{~F}=115.8$; Figure $3 \mathrm{~h} 1$ to $\mathrm{k}$ ). Furthermore, in comparison with animals treated with CHEM alone, animals treated with CHEM + STRESS exhibited reduced levels of new cell production, net neurogenesis, and ongoing neurogenesis ( $p<0.001$, Figure $3 \mathrm{~d}, \mathrm{~g}$, and $\mathrm{k}$ ).

\section{GWIR Chemical-Induced Reduced Neurogenesis Persisted for Prolonged Periods}

Characterization of neurogenesis in the fourth month after the exposure regimen revealed a trend similar to that observed in the immediate postexposure period. Animals exposed to $\mathrm{CHEM}$ or $\mathrm{CHEM}+\mathrm{STRESS}$ exhibited reduced production of new cells per day $(p<0.001, \mathrm{~F}=19.8$; Figure 4 al to $c 2$ and e1), no changes in neuronal fate-choice decision of newly born cells ( $p>0.05, \mathrm{~F}=3.9$; Figure $4 \mathrm{~d} 1$ to $\mathrm{d} 3$ and $\mathrm{e} 2$ ), diminished net neurogenesis $(p<0.001, \mathrm{~F}=21.3$; Figure $3 \mathrm{e} 3)$, and reduced levels of ongoing neurogenesis (based on numbers of DCX + immature neurons, $p<0.0001, \mathrm{~F}=28.3$; Figure 4f1 to i).

Moreover, animals treated with CHEM + STRESS exhibited reduced levels of net neurogenesis and ongoing neurogenesis than animals exposed to CHEM alone ( $p<0.05$, Figure $4 \mathrm{e} 3$ and i).

\footnotetext{
Figure 2 Exposure of adult rats to Gulf war illness-related chemicals results in spatial learning impairments. (al) The bar chart compares average swim speeds of animals treated with vehicle (VEH group), chemicals (CHEM group), and chemicals and stress (CHEM+ STRESS group) during spatial learning. (a2), Learning

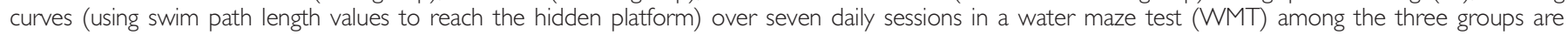

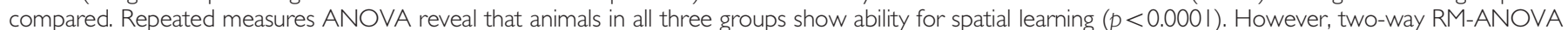

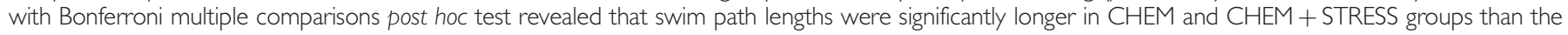

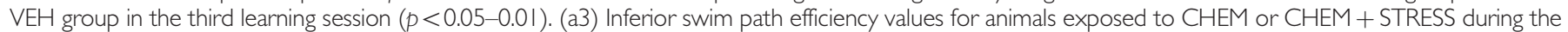

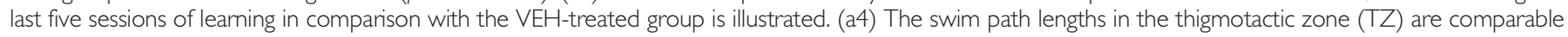

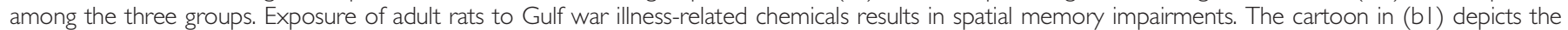

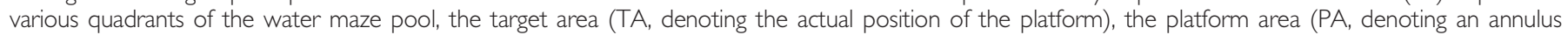

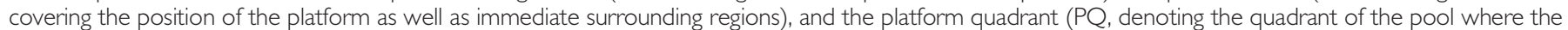

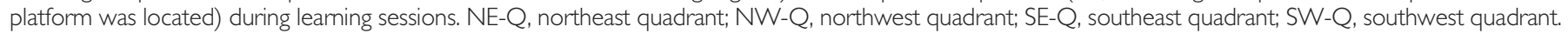

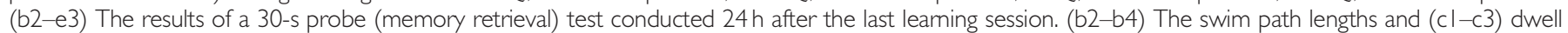

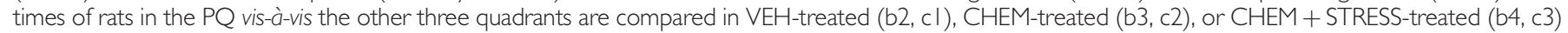

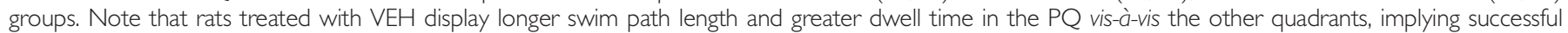

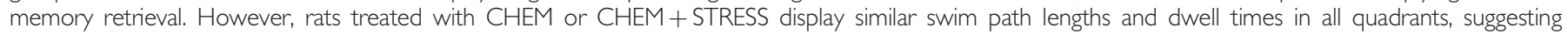

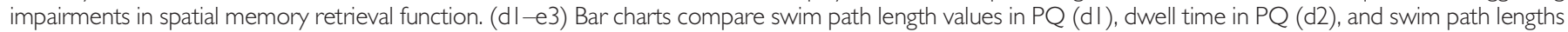
for first entry to TA (el), PA (e2), and PQ (e3) among the three groups. * $p<0.05$; ** $p<0.0$ I; **** $p<0.00$ I; ***** $p<0.000$ I. NS, not significant.
} 

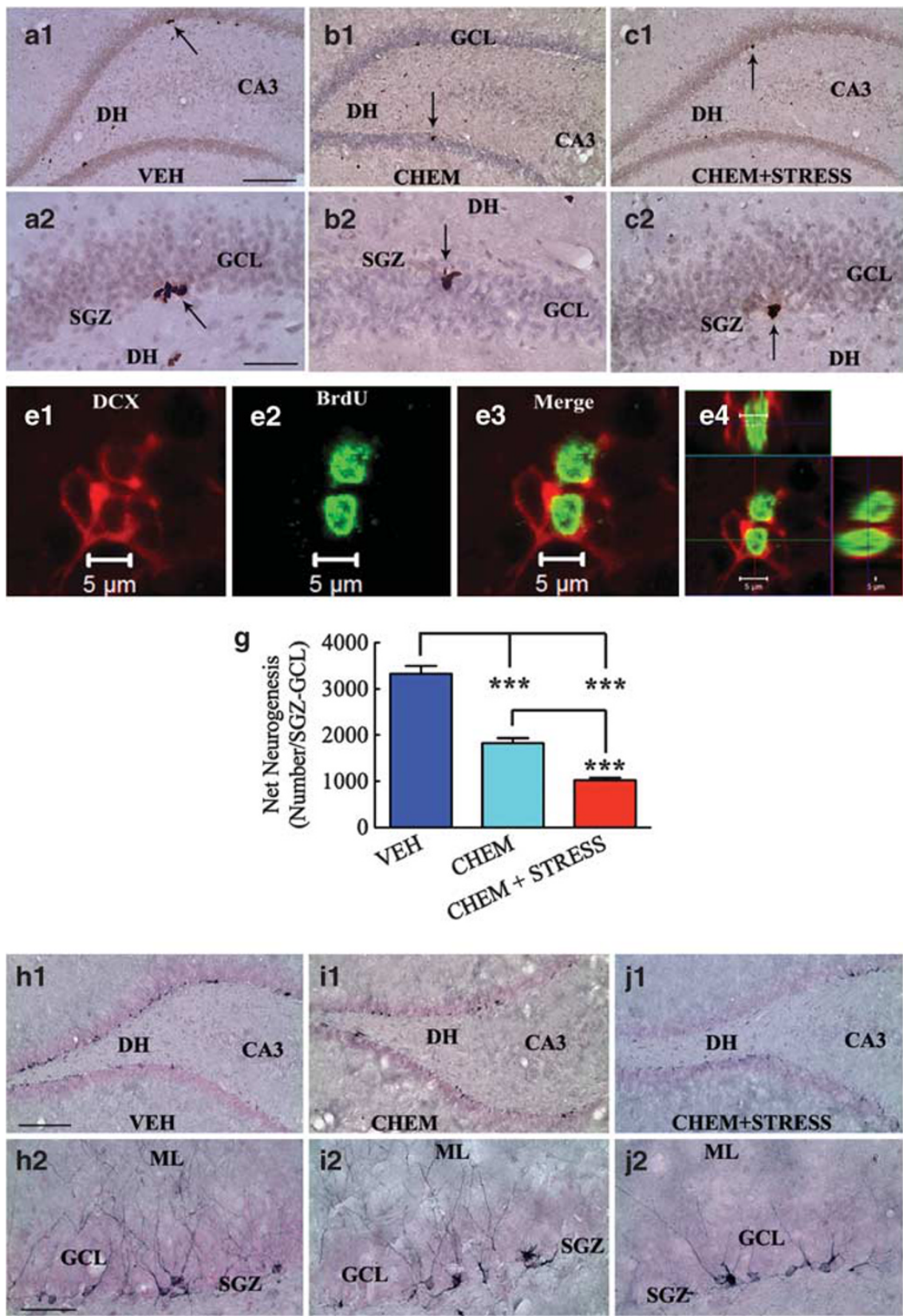
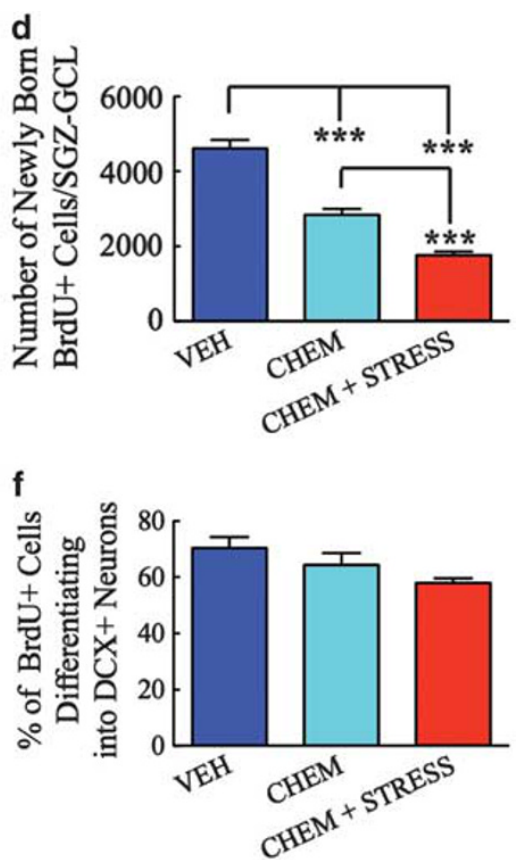

k

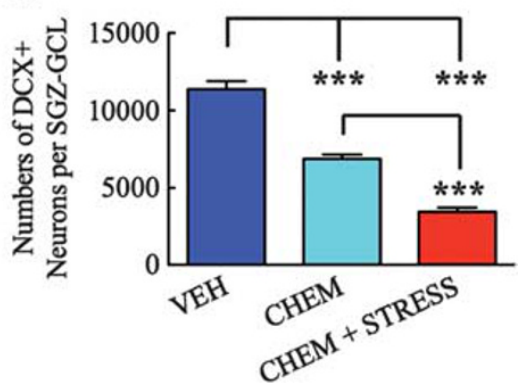

Figure 3 Exposure of adult rats to Gulf war illness-related chemicals reduces hippocampal neurogenesis in the immediate postexposure period, and the addition of mild stress during the period of chemical exposure exacerbates the detrimental effects on neurogenesis. (al-cl) Examples of newly born cells labeled through 5'-bromodeoxyuridine (BrdU) injections (indicated by arrows) in the subgranular zone-granule cell layer (SGZ-GCL) of animals treated with vehicle (VEH, al), chemicals (CHEM, bl), or chemicals and stress (CHEM+STRESS, cl). (a2, b2, c2) Magnified views of some of the BrdU + newly born cells from (al), (bl), and (cl), respectively, are shown. (d) The bar chart shows that the numbers of BrdU + newly born cells in the SGZ-GCL are reduced in animals treated with CHEM or CHEM + STRESS in comparison with VEH-treated control animals. (el-e4) Examples of BrdU + newly born cells that differentiate into doublecortin (DCX)-positive neurons are shown. ( $f$ ) The bar chart shows that percentages of BrdU + newly born cells that differentiate into DCX + neurons are comparable among the three groups. (g) The bar chart shows that net neurogenesis is decreased in animals treated with CHEM or CHEM + STRESS in comparison with VEH-treated control animals. ( $\mathrm{hl}-\mathrm{jl}$ ) The distribution of DCX + newly born neurons between VEH-treated ( $\mathrm{hl}$ ), CHEM-treated (il), and CHEM + STRESS-treated (jl) groups is compared. (h2, i2, j2) Magnified views of some DCX + newly born neurons from (hI), (il), and $(\mathrm{jl})$, respectively, are shown. $(\mathrm{k})$ The bar chart shows that the total number of DCX + newly born neurons is decreased in animals treated with $\mathrm{CHEM}$ or CHEM + STRESS in comparison with VEH-treated animals. DH, dentate hilus; ML, molecular layer. Scale bar: al, bl, cl, hl, il, jl = 200 m; a2, b2, c2, h2, i2, j2 $=50 \mu \mathrm{m} ; \mathrm{el}-\mathrm{e} 4=5 \mu \mathrm{m}$. ***** $\mathrm{p}<0.00 \mathrm{l}$.

\section{Exposure to GWIR Chemicals Did Not Kill SGZ-NSCs But Inhibited Their Proliferation}

Immunohistochemistry for Sox-2 suggested normal distribution (density) of Sox- $2+$ cells in the SGZ of all groups (Figure 5a1 to a3). Dual immunofluorescence analyses for
Sox-2 and S- $100 \beta$ demonstrated comparable distribution of Sox- $2+$ cells expressing S-100 $\beta$ (Sox- $2+$ mature astrocytes) and Sox- + cells lacking S-100 $\beta$ (Sox-2 + putative NSCs) in all groups (Figure 5b1 to b3). Furthermore, total numbers of Sox- $2+$ cells and Sox- $2+$ putative NSCs in the 
SGZ were comparable across the three groups $(p>0.05$, $\mathrm{F}=1.8-4.2$; Figure $5 \mathrm{c} 1$ and $\mathrm{c} 2$ ). However, analyses of the percentage of Sox- $2+$ cells that express Ki-67 (a marker of dividing cells, Figure $5 \mathrm{~d} 1$ to e3) in the SGZ revealed fewer NSCs undergoing proliferation in animals exposed to CHEM or CHEM + STRESS in comparison with their
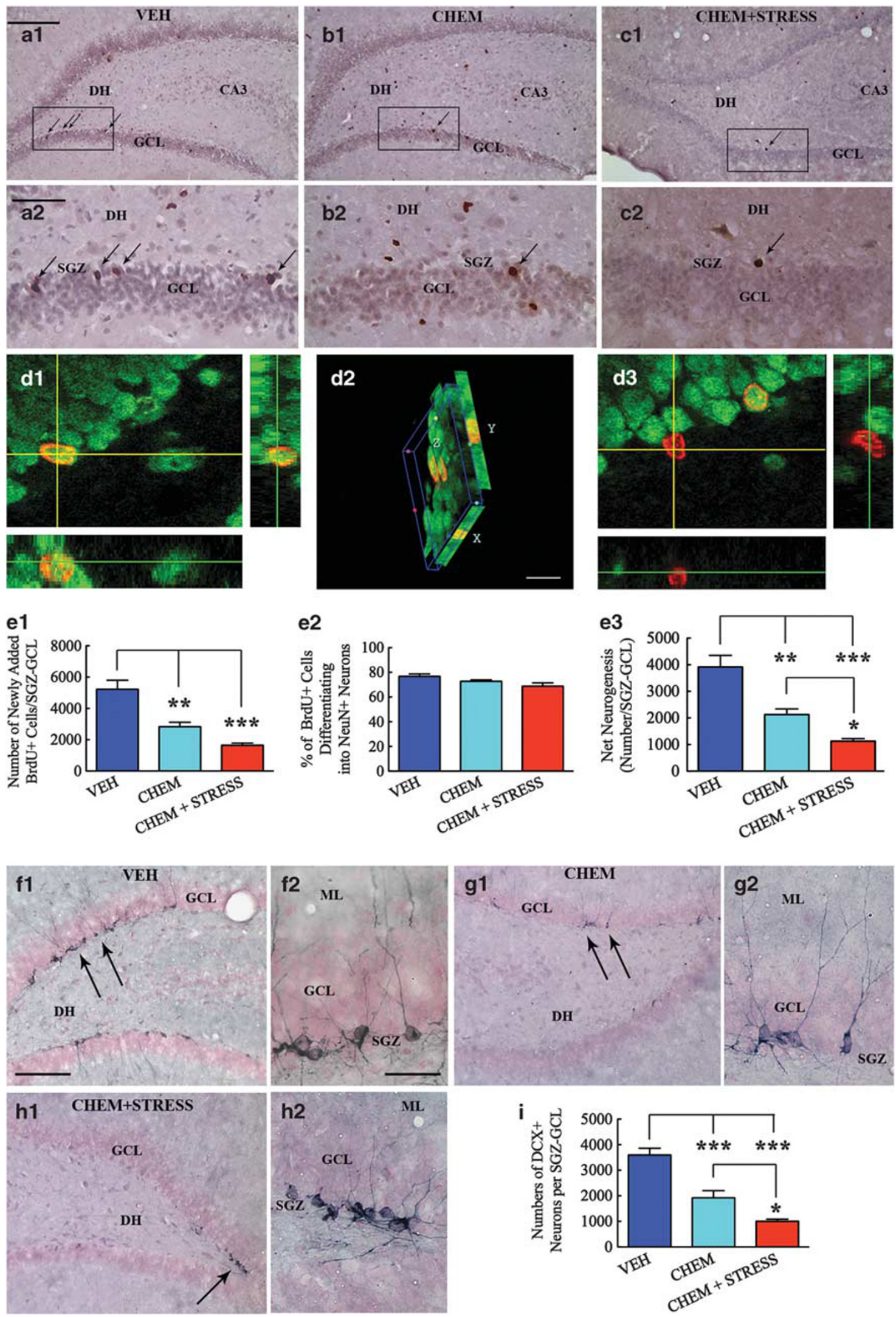

Figure 4 For caption please refer page 2356. 
counterparts in VEH-treated animals $(p<0.05, \mathrm{~F}=5.97$, Figure 5f1; $p<0.0001, \mathrm{~F}=142.1$; Figure $5 \mathrm{f} 2$ ). The overall detrimental effect on proliferation was, however, similar between animals treated with CHEM or CHEM + STRESS.

\section{Exposure to GWIR Chemicals Induced Hippocampal Neuron Loss}

Examination of NeuN-immunostained tissue sections revealed normal hippocampal cytoarchitecture in all groups (Figure 6a1 to c4). However, stereological quantification demonstrated partial loss of neurons in several cell layers (GCL and DH of the DG, and CA1 cell layer) of animals exposed to CHEM or CHEM + STRESS $(p<0.05-0.001$, $\mathrm{F}=5.2-15.5$; Figure $6 \mathrm{~d} 1$ to $\mathrm{d} 3$ ). However, the overall loss was similar between animals treated with CHEM or CHEM + STRESS except for the CA3 cell layer where only animals exposed to CHEM + STRESS showed loss of neurons $(p<0.01$, Figure $6 \mathrm{~d} 4)$. Furthermore, exposure to GWIR chemicals decreased volumes of the DG (significant for CHEM + STRESS group, $p<0.05, F=4.4$; Figure 6e1) and the CA3 subfield (significant for both CHEM and CHEM + STRESS groups, $p<0.05, \mathrm{~F}=5.3$; Figure 6e3) but not the CA1 subfield $(p>0.05, F=2.9$; Figure 6e2). However, the overall hippocampal volume including fimbria was significantly decreased in both CHEM and CHEM + STRESS groups $(p<0.01, \mathrm{~F}=6.6$; Figure 6e4).

\section{Exposure to GWIR Chemicals Resulted in Mild Hippocampal Inflammation}

Analyses of sections using ED-1 antibody demonstrated the presence of activated microglia in the hippocampus of animals treated with CHEM or CHEM + STRESS (Figure $7 \mathrm{~b} 1$ to $\mathrm{c} 4$ ). Interestingly, these microglia were frequently observed in the hippocampal fissure (Figure $7 \mathrm{~b} 2$ and $\mathrm{c} 1$ ) and fimbria (Figure 7b1, c2, and c3). However, some isolated microglia could also be seen in the CA1 stratus radiatum (Figure $7 \mathrm{c} 4$ ). The overall density of activated microglia was greater in animals exposed to CHEM + STRESS than animals exposed to CHEM alone $(p<0.05$, Figure $7 \mathrm{~d}$ ). Analyses using nestin and vimentin antibodies did not reveal the presence of reactive astrocytes in animals exposed to GWIR chemicals. However, GFAP immunostaining demonstrated astrocytes exhibiting hypertrophy in animals treated with CHEM (Figure 7f1 to f3) or CHEM + STRESS (Figure 7g1 to g3) in comparison with VEH-treated animals (Figure $7 \mathrm{e} 1$ to e3). Area fraction analyses of GFAP + structures revealed that animals exposed to CHEM or CHEM + STRESS exhibited hypertrophy of GFAP + structures in all regions of the hippocampus in comparison with the VEH group $(p<0.01-0.0001, \quad \mathrm{~F}=12.6-43.8$; Figure $7 \mathrm{~h} 1$ to $\mathrm{h} 4, p<0.01-0.001)$. However, area fractions of GFAP + structures were comparable between CHEM and CHEM + STRESS groups.

\section{Exposure of NSCs to DEET and/or PM Inhibited NSC Proliferation In Vitro}

In control cultures, $\sim 7 \%$ of plated cells proliferated and formed clear larger neurospheres. Interestingly, the presence of $100 \mu \mathrm{M}$ PB did not interfere with NSC proliferation, as both number and size of neurospheres were comparable between control and PB-treated cultures (Supplementary Figure S3a1, a2, and b). However, the presence of $100 \mu \mathrm{M}$ DEET or PM considerably decreased the proliferation of NSCs (Supplementary Figure S3a3 and a4). This was evidenced by $76-78 \%$ reduction in the numbers of neurospheres generated per 100 cells plated (Supplementary Figure S3b) and reduced size of neurospheres (Supplementary Figure S3a3 and a4). Furthermore, a combination of PB, DEET, and PM decreased neurosphere formation by 92\% (Supplementary Figure S3a5 and b).

\section{DISCUSSION}

This study provides novel evidence that combined exposure to low doses of GWIR chemicals for 4 weeks is sufficient for inducing chronic hippocampal dysfunction typified by increased depressive- and anxiety-like behavior, and spatial learning and memory impairments in a rat model. These findings are akin to depression, anxiety, and cognitive dysfunction reported in veterans with GWI (Haley et al, 1997; David et al, 2002; Li et al, 2011; Odegard et al, 2012). At the structural level, these impairments were associated with persistent reductions in dentate neurogenesis, partial loss of principal neurons, mild neuroinflammation, and reduced hippocampal volume. Moreover, addition of mild stress in the period of exposure to GWIR chemicals exacerbated mood, cognitive, and neurogenesis impairments, although application of mild stress alone has been shown to be associated with beneficial effects on cognitive and mood function (Parihar et al, 2011). In vitro analyses

Figure 4 Exposure of adult rats to Gulf war illness-related chemicals reduces hippocampal neurogenesis even at 4 months after the exposure and the

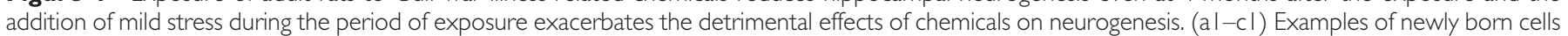

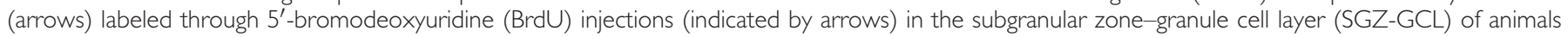

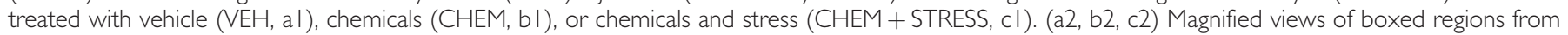

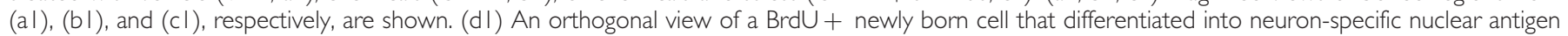

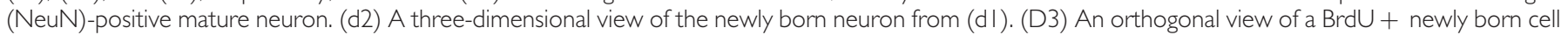

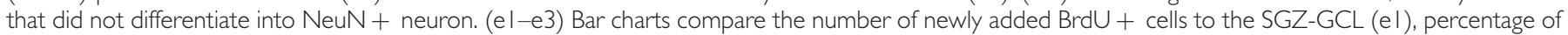

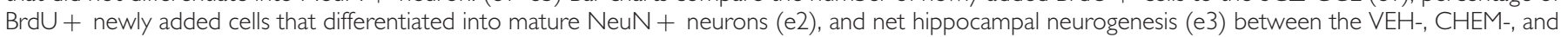

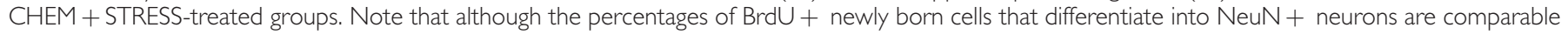

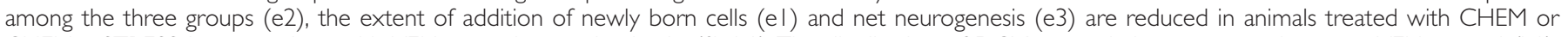

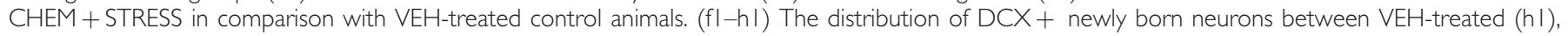

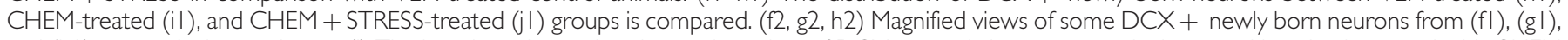

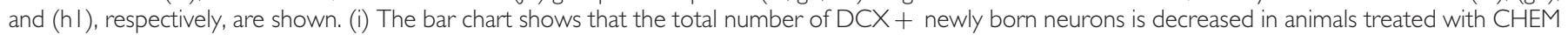

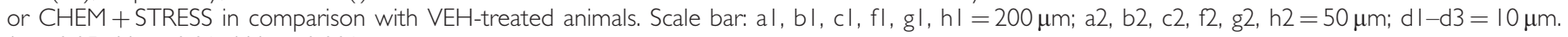
* $p<0.05$; ** $p<0.01$; **** $p<0.001$. 

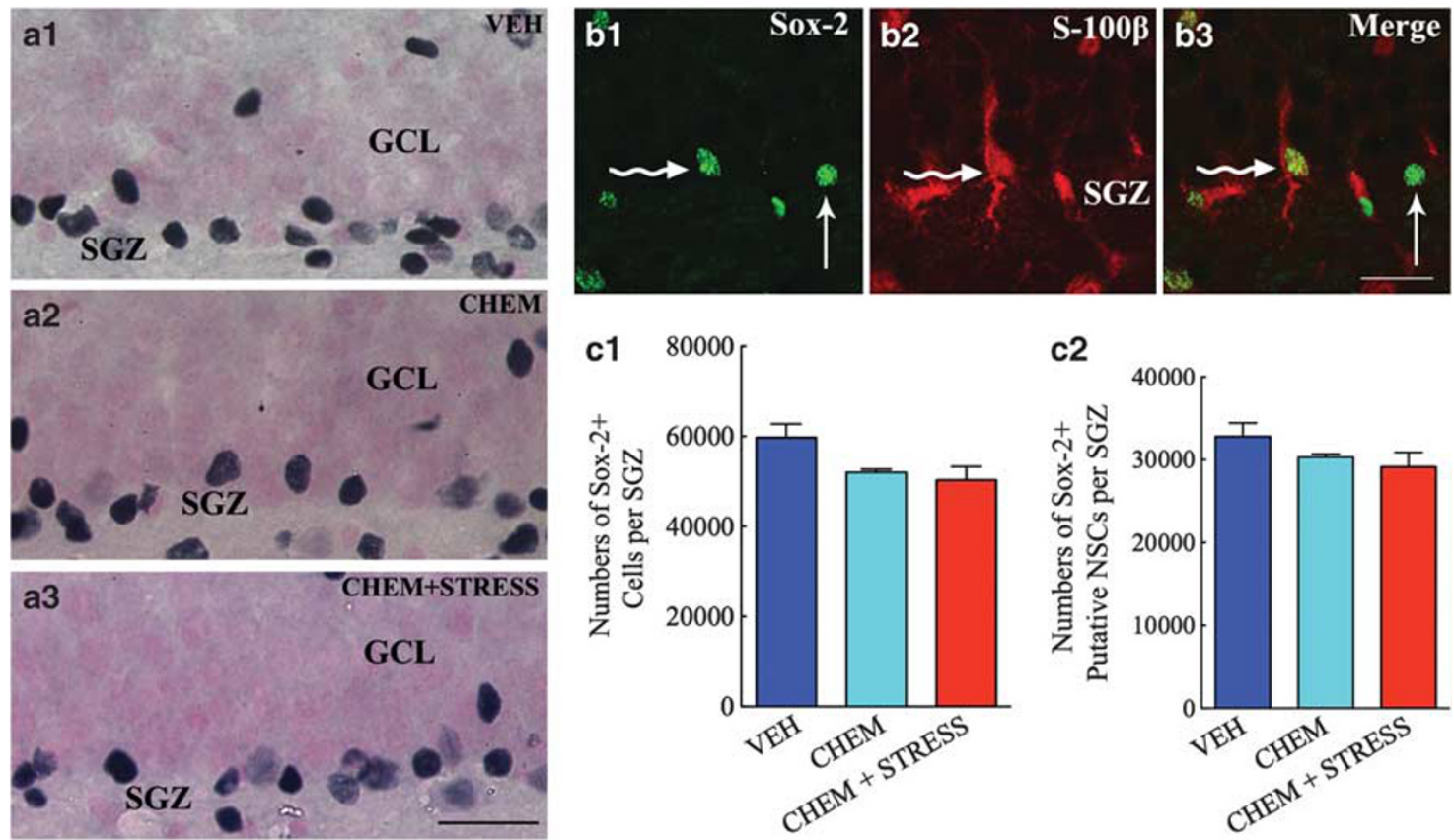
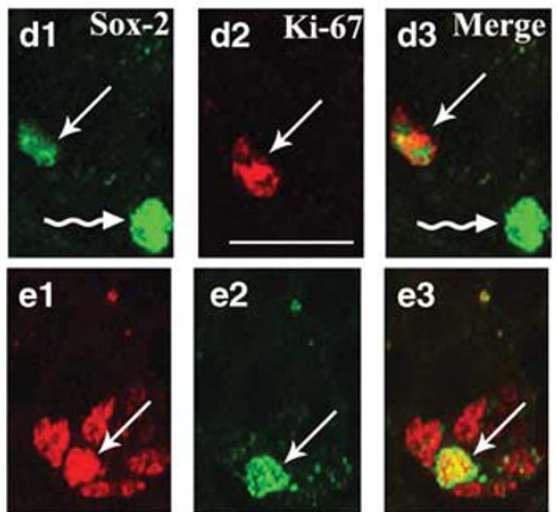

f1
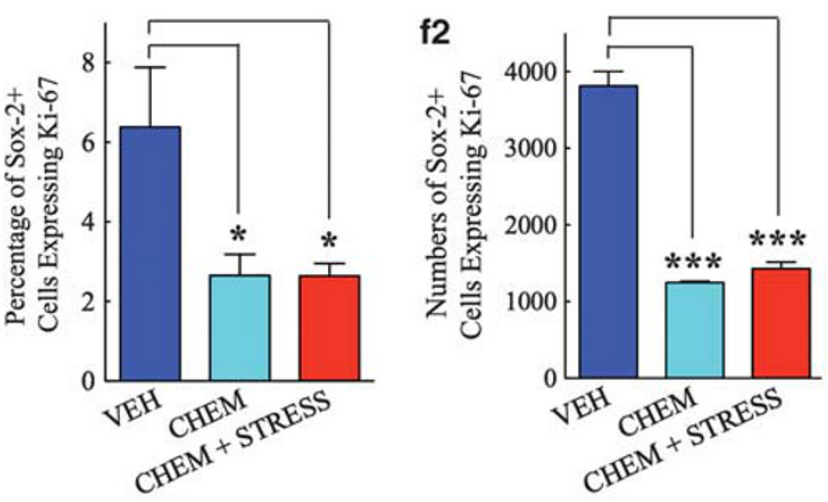

Figure 5 Exposure of adult rats to Gulf war illness-related chemicals or chemicals and stress does not kill neural stem cells (NSCs) but induce alterations in NSC proliferative behavior. (al-a3) The distribution of Sox-2 + cells in the subgranular zone-granule cell layer (SGZ-GCL) of animals treated with vehicle (VEH, al), chemicals (CHEM, a2), or chemicals and stress (CHEM + STRESS, a3) is shown. (bI-b3) Examples of Sox-2 + cells that lack S-I00 $\beta$ (putative NSCs, indicated by arrows) and Sox-2 + cells that express S-100 $\beta$ (mature astrocytes, indicated by curved arrows) in the SGZ. (cl, c2) Bar charts demonstrate that numbers of both Sox-2 + cells (cl) and Sox-2+ cells that lack S-100 $\beta$ (putative NSCs; 2 ) are comparable among the three groups in the SGZ. (dI-e3) Examples of Sox-2 + cells expressing Ki-67 (ie, proliferating NSCs, indicated by arrows) in the SGZ. Curved arrows show Sox-2 + cells that lack Ki-67 expression. (fI, f2) Bar charts show that animals treated with CHEM or CHEM+STRESS display reduced percentages as well as numbers of Sox-2 + cells

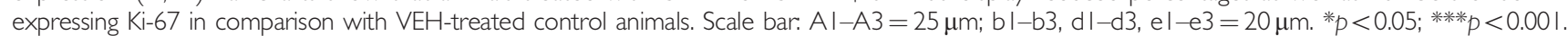

further showed that PM and DEET (but not PB) exposure can greatly inhibit the proliferation of NSCs. Interestingly, cognitive dysfunction observed in this rat model contrasts with a recent study in C57BL/6 mouse model where exposure to similar doses of chemicals and stress did not impair cognitive function but enhanced anxiety when examined 20 days after the exposure (Abdullah et al, 2012). The discrepancy between the two studies likely reflects differences in animal species and timing of behavioral analyses.

\section{Links Between Mood and Cognitive Dysfunction and Impaired Hippocampal Neurogenesis}

Although multiple factors can interfere with mood and cognitive function, changes in the quantity of adult hippocampal neurogenesis are widely believed to influence both mood and spatial learning and memory function (Deng et al, 2010; Eisch and Petrik, 2012). Indeed, we found substantial decreases in hippocampal neurogenesis at both early and extended periods after the exposure to GWIR chemicals. In addition, reduced neurogenesis was associated with decreased volume of the hippocampus. Considering these, a question emerges of whether persistently reduced hippocampal neurogenesis is one of the underlying causes of mood and memory dysfunction in GWI. This proposition is supported by multiple observations in previous studies. First, there are credible evidences in favor of the hypothesis that mood function depends on the extent of hippocampal neurogenesis (Snyder et al, 2011; Eisch and Petrik, 2012; Kheirbek et al, 2012), which includes findings that humans with depression exhibit smaller hippocampal volume likely 

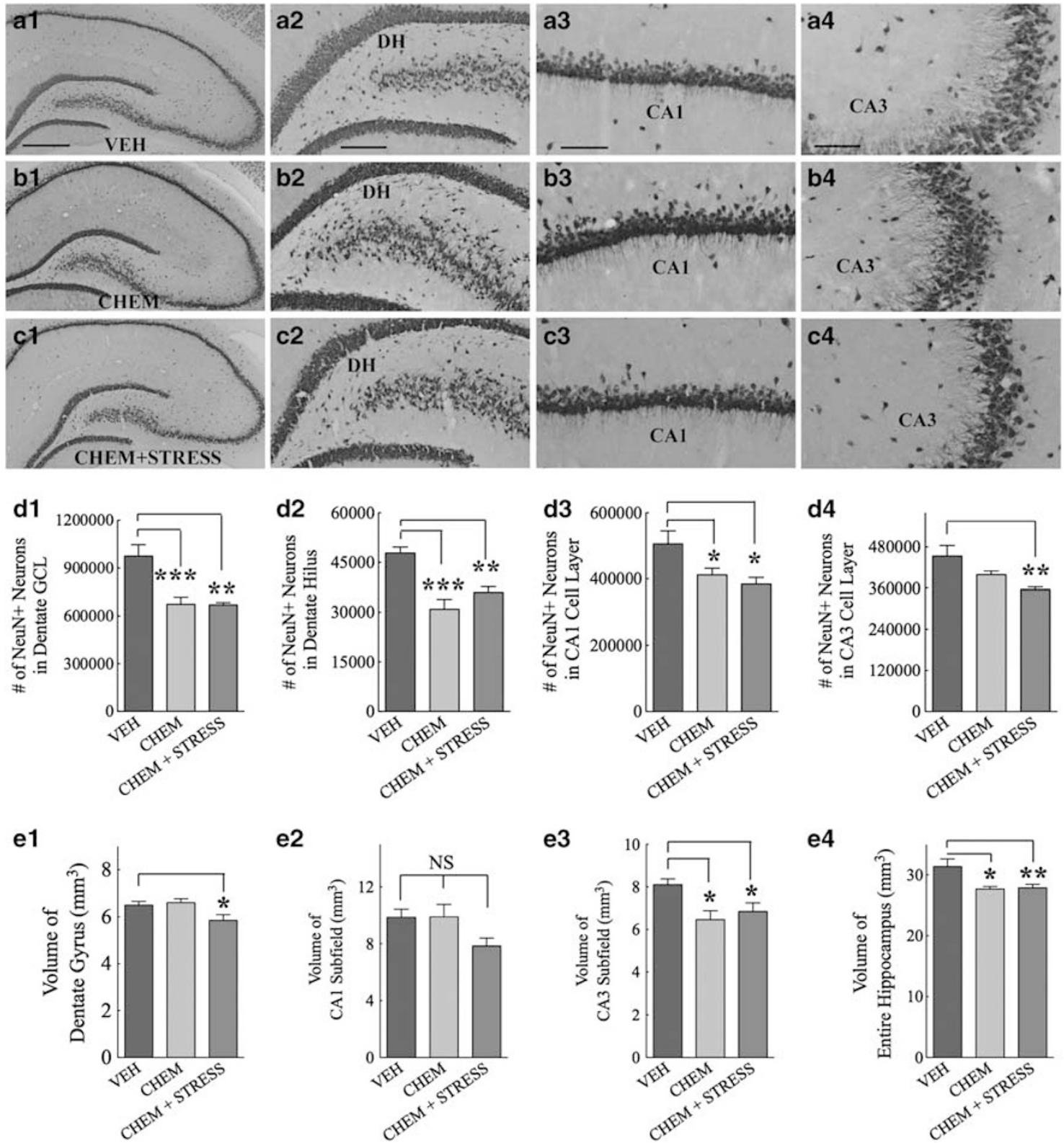

Figure 6 Exposure of adult rats to Gulf war illness-related chemicals or chemicals and stress induces partial neuron loss in the hippocampus. (al, bl, cl) The distribution of neuron-specific nuclear antigen ( $\mathrm{NeuN}$ )-positive neurons in different layers of the hippocampus of animals treated with vehicle (VEH, a I), chemicals (CHEM, bl), or chemicals and stress (CHEM + STRESS, cl). (a2-c4) Magnified views of regions of the dentate gyrus (a2, b2, c2), CAI subfield (a3, b3, c3), and CA3 subfield from (al), (bl), and ( $\mathrm{cl})$. ( $\mathrm{dl}-\mathrm{d} 4)$ Bar charts demonstrate that animals treated with CHEM or CHEM + STRESS display some loss of neurons in the granule cell layer (dI), dentate hilus (d2), CAI pyramidal cell layer (d3), and CA3 pyramidal cell layer (d4) in comparison with VEH-treated control animals. (el-e4) Bar charts compare volumes of the dentate gyrus (el), CAI subfield (e2), CA3 subfield (e3), and the entire hippocampus including

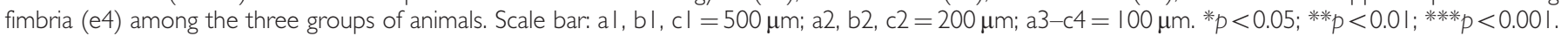

because of persistently reduced neurogenesis (Small et al, 2011; Fotuhi et al, 2012), antidepressants upregulate hippocampal neurogenesis with a lag time comparable with the delay between antidepressant treatment and positive clinical outcome on mood function (Malberg et al, 2000), and ablation of hippocampal neurogenesis impairs antidepressant efficacy (Santarelli et al, 2003). Although a few rodent studies have contradicted the above association (Meshi et al, 2006; David et al, 2009), there is considerable support in favor of the neurogenic hypothesis of depression, which includes a recent non-human primate study showing that hippocampal neurogenesis is necessary for the therapeutic action of antidepressants (Perera et al, 2011; Eisch and Petrik, 2012). Second, although a negative correlation was seen in a few earlier studies (Bizon et al, 2004), most studies support an important role for hippocampal neurogenesis in spatial memory function. This includes observations that animals housed in enriched environment, undergoing physical exercise, treated for corticosterone reductions, or exposed to predictable chronic mild stress displayed enhanced neurogenesis and improved spatial memory function (Kempermann et al, 2002; van Praag et al, 2005; Montaron et al, 2006; Parihar et al, 2011), and aged rats with unimpaired spatial memory 

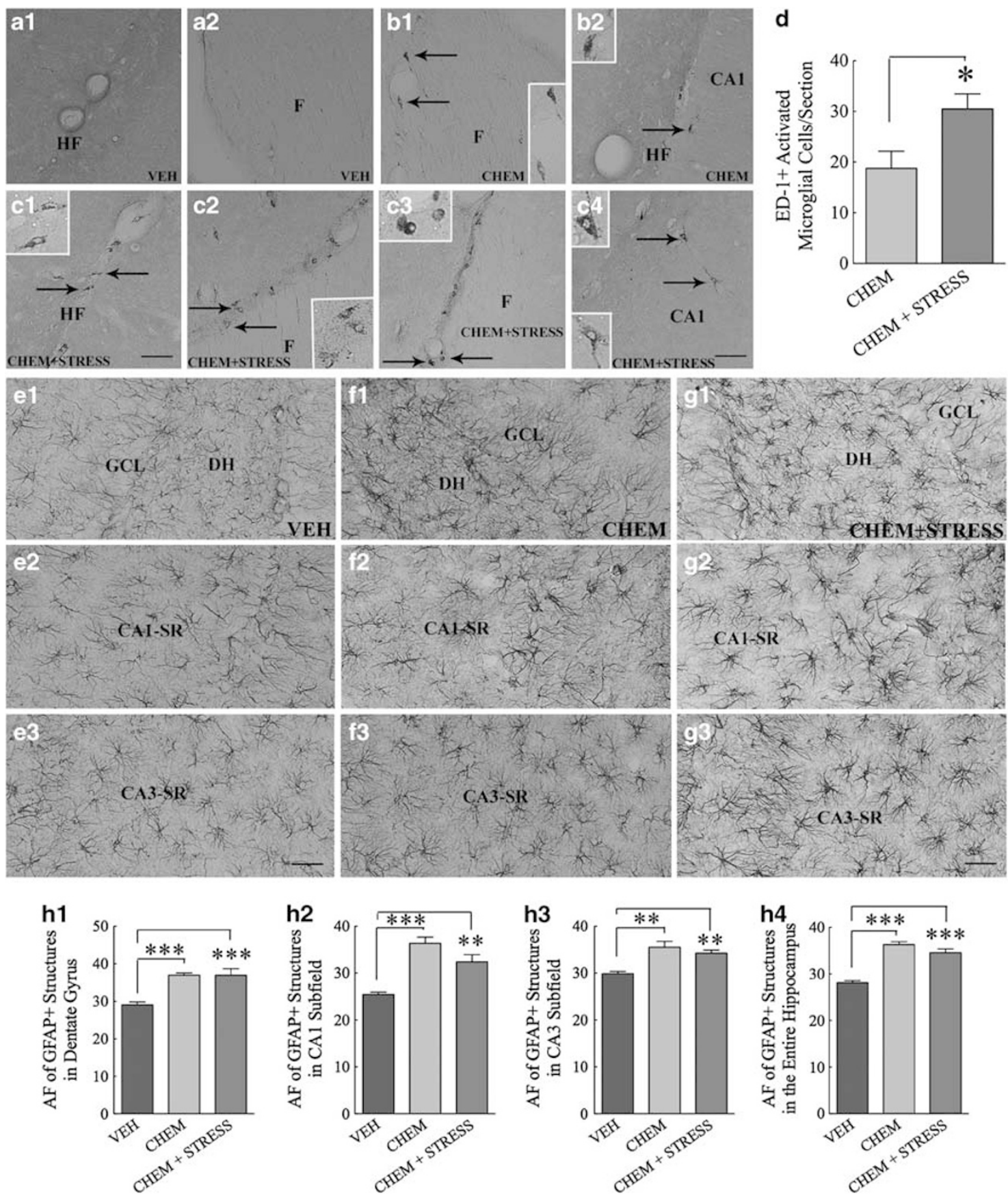

Figure 7 Exposure of adult rats to Gulf war illness-related chemicals or chemicals and stress induces microglial and astrocyte reactivity in the hippocampus. (a $\mathrm{I}-\mathrm{c} 4)$ The occurrence of ED-I + activated microglia in regions such as fimbria (F) and the hippocampal fissure (HF) in animals treated with CHEM (bl, b2) or CHEM + STRESS ( $\mathrm{cl}-\mathrm{c} 4)$ in contrast to their absence in animals treated with VEH (al, a2). (bl-c4) Inserts illustrate the magnified view of the morphology of activated microglia indicated by arrows. (d) The bar chart compares the number of ED-I + activated microglia between CHEM and CHEM + STRESS groups. (el-g3) The distribution and morphology of GFAP + astrocytes in the dentate gyrus (upper row), CAI subfield (middle row), and CA3 subfield (lower row) between animals treated with VEH (el-e3), CHEM (fl-f3) or CHEM+STRESS (gl-g3) is compared. Note the presence of hypertrophied GFAP + astrocytes in hippocampal regions of animals treated with CHEM (fI-f3) or CHEM + STRESS (gI-g3) but not in animals treated with VEH (el-e3). Scale bar: el-g3 = I00 $\mu \mathrm{m}$. (hl-h4) Bar charts compare area fraction of GFAP + structures among the three groups in the dentate gyrus (hI), CAI subfield (h2), CA3 subfield (h3), and the entire hippocampus (h4). $* p<0.05$; $* * * 0.01$; $* * * *<0.00$ I. DH, dentate hilus; GCL, granule cell layer; CAI-SR, CAI stratum radiatum; CA3-SR, CA3 stratum radiatum.

ability displayed greater extent of neurogenesis than rats with impaired spatial memory (Drapeau et al, 2003). Moreover, recent studies that utilized selective neurogenesis ablation techniques strongly support a role for hippocampal neurogenesis in spatial memory function (Dupret et al, 2007, 2008; Wang et al, 2012). Interestingly, animals exposed to GWIR chemicals did not display impaired recognition memory function in NORT, which may be because of a partial involvement of the hippocampus in this task (Broadbent et al, 2009).

Thus, reduced hippocampal neurogenesis likely contributes to mood and cognitive dysfunction observed in GWI. 
Although the basic in vivo imaging correlates of human hippocampal neurogenesis (such as quantification of cerebral blood flow in the SGZ) is feasible (Manganas et al, 2007; Pereira et al, 2007), greater advances in imaging techniques will be required to correlate the loss of neurogenesis with altered mood or cognitive function in veterans with GWI. However, several previous studies have shown the presence of hippocampal dysfunction as well as increased incidence of depression, anxiety, and memory dysfunction in GW veterans (David et al, 2002; Black et al, 2004; Menon et al, 2004; Li et al, 2011; Odegard et al, 2012). MRI studies have also shown smaller hippocampus in a subset of GW veterans exhibiting posttraumatic stress disorder but not in GW veterans affected with depression alone (Vythilingam et al, 2005; Apfel et al, 2011).

\section{Other Factors Contributing to GWIR Chemical-Mediated Hippocampal Dysfunction}

Quantification of NeuN + neurons revealed 19-31\% loss of neurons in different subfields of the hippocampus, with no differences in the extent of neuron loss between animals exposed to CHEM or CHEM + STRESS. Additional analyses suggested mild inflammation typified by the occurrence of activated microglia in white matter regions and hypertrophy of astrocytes in all subfields. Although previous studies have suggested some neuron loss in the hippocampus with exposure to CHEM + STRESS (Abdel-Rahman et al, 2002, 2004a), occurrences of neuron loss, activated microglia, and hypertrophied astrocytes in animals exposed to CHEM alone are additional novel findings in this study. Neurodegeneration is likely because of both direct and indirect effects of chemicals. For instance, PM can mediate direct neurodegeneration by causing prolonged activation of voltage-gated sodium channels and hyperexcitability in neurons (Ray and Fry, 2006). On the other hand, DEET is a weak AChE inhibitor but can enhance the activity of other AChE inhibitors such as PB (Corbel et al, 2009). Previous studies in this GWI model have indeed seen both decreased AChE activity and disruption of the blood brain barrier in multiple brain regions (Abdel-Rahman et al, 2002, 2004a). Because both partial neurodegeneration and inflammation can interfere with hippocampal function and neurogenesis (Krishnadas and Cavanagh, 2012; Kohman and Rhodes, 2013), it is plausible that these two factors have also contributed to mood and cognitive dysfunction and impaired neurogenesis observed in animals exposed to GWIR chemicals. Additional factors may also be involved. For example, a recent lipidomics study reported that regulation of acetylcholine regulator and signaling pathways undergo alterations with exposure to GWIR chemicals (Abdullah et al, 2012).

\section{Causes of Reduced Hippocampal Neurogenesis in Animals Exposed to GWIR Chemicals}

Our analyses revealed that similar numbers of putative NSCs persist, and comparable fractions of newly born cells differentiate into mature neurons between VEH-treated animals and animals treated with GWIR chemicals. This suggested that exposure to low doses of GWIR chemicals did not kill NSCs or alter the neuronal fate-choice decision of new cells generated by NSCs. However, fewer NSCs were undergoing proliferation in animals exposed to GWIR chemicals. These results imply that reduced proliferation of NSCs is the underlying cause of declined neurogenesis in animals exposed to GWIR chemicals. Reduced proliferation of NSCs in the exposure period can result from direct effects of chemicals, as our in vitro assays showed that DEET and PM can greatly inhibit NSC proliferation at $\mu \mathrm{M}$ concentrations. On the other hand, reduced proliferation of NSCs observed in the fifth month after the exposure is likely because of changes in NSC microenvironment following exposure to chemicals. Apart from the mild inflammation noted above, this may also involve increased oxidative stress observed in previous studies with exposure to GWIR chemicals (Li et al, 2001; Abu-Qare et al, 2001). Thus, inflammation and oxidative stress mediated by GWIR chemicals are the likely causes of NSC dysfunction in GWI.

\section{CONCLUSIONS}

We show the first evidence of an association between mood and cognitive dysfunction and hippocampal pathology typified by decreased neurogenesis, partial loss of principal neurons, and mild inflammation in a rat model of GWI. Hence, application of novel treatment strategies that are efficacious for enhancing neurogenesis as well as suppressing inflammation may be helpful for improving mood and cognitive function in veterans with GWI.

\section{FUNDING AND DISCLOSURE}

The authors declare no conflict of interest.

\section{ACKNOWLEDGEMENTS}

This study was supported primarily by a grant for 'Gulf War Illness Research' from the Department of Veterans Affairs (Merit Review Award to AKS) and partly by a grant from the Texas A\&M Health Science Center (Emerging Technology Funds to AKS).

\section{Author contributions}

VKP, BH, and AKS contributed equally to this work. VKP performed chemical and stress exposures to animals, behavioral experiments, immunohistochemistry, stereological cell counts, collection, analyses, and interpretation of data and prepared an initial rough draft of the manuscript text. BH gave input to behavioral experiments, performed immunohistochemistry, stereological cell counts, confocal microscopy, and contributed to collection, analyses, and interpretation of behavioral data and assembly of figures. BS performed chemical and stress exposures to animals, tissue processing, histology, immunohistochemistry, and contributed to behavioral experiments and collection of data. AKS conceived the study, conceptualized the experimental design, analyzed, interpreted and assembled data, prepared all figures and wrote the manuscript. All authors gave input to the manuscript text and approved the final version of the manuscript. 


\section{REFERENCES}

Abdel-Rahman A, Shetty AK, Abou-Donia MB (2002). Disruption of the blood-brain barrier and neuronal cell death in cingulate cortex, dentate gyrus, thalamus, and hypothalamus in a rat model of Gulf-War syndrome. Neurobiol Dis 10: 306-326.

Abdel-Rahman A, Abou-Donia S, El-Masry E, Shetty A, AbouDonia M (2004a). Stress and combined exposure to low doses of $\mathrm{PB}, \mathrm{DEET}$, and permethrin produce neurochemical and neuropathological alterations in cerebral cortex, hippocampus, and cerebellum. J Toxicol Environ Health A 67: 163-192.

Abdel-Rahman A, Rao MS, Shetty AK (2004b). Nestin expression in hippocampal astrocytes after injury depends on the age. Glia 47: 299-313.

Abdullah L, Crynen G, Reed J, Bishop A, Phillips J, Ferguson S et al (2011). Proteomic CNS profile of delayed cognitive impairment in mice exposed to Gulf War agents. Neuromolecular Med 13: 275-288.

Abdullah L, Evans JE, Bishop A, Reed JM, Crynen G, Phillips J et al (2012). Lipidomic profiling of phosphocholine containing brain lipids in mice with sensorimotor deficits and anxiety-like features after exposure to gulf war agents. Neuromolecular Med 14: 349-361.

Abu-Qare W, Suliman HB, Abou-Donia MB (2001). Induction of urinary excretion of 3-nitrotyrosine, a marker of oxidative stress, following administration of pyridostigmine bromide, DEET (N,N-diethyl-m-toluamide) and permethrin, alone and in combination in rats. Toxicol Lett 121: 127-134.

Amato AA, McVey A, Cha C, Matthews EC, Jackson CE, Kleingunther $\mathrm{R}$ et al (1997). Evaluation of neuromuscular symptoms in veterans of the Persian Gulf War. Neurology 48: $4-12$.

Apfel BA, Ross J, Hlavin J, Meyerhoff DJ, Metzler TJ, Marmar CR et al (2011). Hippocampal volume differences in Gulf War veterans with current versus lifetime posttraumatic stress disorder symptoms. Biol Psychiatry 69: 541-548.

Bilbo SD, Smith SH, Schwarz JM (2012). A lifespan approach to neuroinflammatory and cognitive disorders: a critical role of glia. J Neuroimmune Pharmacol 7: 24-41.

Binns JH, Barlow C, Bloom FE, Clauw DJ, Golomb BA, Graves JC et al (2008). Gulf war illness and the health of Gulf war veterans: scientific findings and recommendations, Research Advisory Committee Report on Gulf War Illness and Health of Gulf War Veterans. Dept of Veterans Affairs. US Government Printing Office: Washington, DC, pp 1-465.

Bizon JL, Lee HJ, Gallagher M (2004). Neurogenesis in a rat model of age-related cognitive decline. Aging Cell 3: 227-234.

Black DW, Carney CP, Peloso PM, Woolson RF, Schwartz DA, Voelker MD, Barrett DH, Doebbeling BN (2004). Gulf War veterans with anxiety: prevalence, co-morbidity, and risk factors. Epidemiology 15: 135-142.

Broadbent NJ, Gaskin S, Squire LR, Clark RE (2009). Object recognition memory and the rodent hippocampus. Learn Mem 17: 5-11.

Chaney LA, Wineman RW, Rockhold RW, Hume AS (2000). Acute effects of an insect repellent, N,N-diethyl-m-toluamide, on cholinesterase inhibition induced by pyridostigmine bromide in rats. Toxicol Appl Pharmacol 165: 107-114.

Corbel V, Stankiewicz M, Pennetier C, Fournier D, Stojan J, Girard E et al (2009). Evidence for inhibition of cholinesterases in insect and mammalian nervous systems by the insect repellent DEET. BMC Biol 7: 47.

David AS, Farrin L, Hull L, Unwin C, Wessely S, Wykes T (2002). Cognitive functioning and disturbances of mood in UK veterans of the Persian Gulf War: a comparative study. Psychol Med 32: 1357-1370.

David DJ, Samuels BA, Rainer Q, Wang JW, Marsteller D, Mendez I et al (2009). Neurogenesis-dependent and-independent effects of fluoxetine in an animal model of anxiety/depression. Neuron 62: 479-493.

Deng W, Aimone JB, Gage FH (2010). New neurons and new memories: how does adult hippocampal neurogenesis affect learning and memory? Nat Rev Neurosci 11: 339-350.

Drapeau E, Mayo W, Aurousseau C, Le Moal M, Piazza PV, Abrous DN (2003). Spatial memory performances of aged rats in the water maze predict levels of hippocampal neurogenesis. Proc Natl Acad Sci USA 100: 14385-14390.

Dupret D, Fabre A, Döbrössy MD, Panatier A, Rodríguez JJ, Lamarque $S$ et al (2007). Spatial learning depends on both the addition and removal of new hippocampal neurons. PLoS Biol 5: e214.

Dupret D, Revest JM, Koehl M, Ichas F, De Giorgi F, Costet P et al (2008). Spatial relational memory requires hippocampal adult neurogenesis. PLoS One 3: e1959.

Eisch AJ, Petrik D (2012). Depression and hippocampal neurogenesis: a road to remission? Science 338: 72-75.

Everson MP, Kotler S, Blackburn WD (1999). Stress and immune dysfunction in Gulf War veterans. Ann NY Acad Sci 876: 413-418.

Fotuhi M, Do D, Jack C (2012). Modifiable factors that alter the size of the hippocampus with ageing. Nat Rev Neurol 8: 189-202.

Friedman A, Kaufer D, Shemer J, Hendler I, Soreq H, Tur-Kaspa I (1996). Pyridostigmine brain penetration under stress enhances neuronal excitability and induces early immediate transcriptional response. Nat Med 2: 1382-1385.

Golomb BA (2008). Acetylcholinesterase inhibitors and Gulf War illnesses. Proc Natl Sci Acad Sci USA 105: 4295-4300.

Haley RW, Kurt TL (1997). Self-reported exposure to neurotoxic chemical combinations in the Gulf war: a cross-sectional epidemiologic study. JAMA 277: 231-237.

Haley RW, Hom J, Roland PS, Bryan WW, Van Ness PC, Bonte FJ et al (1997). Evaluation of neurologic function in Gulf War veterans: a blinded case-control study. JAMA 277: 223-230.

Haley RW, Fleckenstein JL, Marshall WW, McDonald GG, Kramer GL, Petty F (2000a). Effect of basal ganglia injury on central dopamine activity in Gulf War syndrome: correlation of proton magnetic resonance spectroscopy and plasma homovanillic acid levels. Arch Neurol 57: 1280-1285.

Haley RW, Marshall WW, McDonald GG, Daugherty MA, Petty F, Fleckenstein JL (2000b). Brain abnormalities in Gulf War syndrome: evaluation with $1 \mathrm{H}$ MR spectroscopy. Radiology 215: 807-817.

Hattiangady B, Rao MS, Shetty AK (2004). Chronic temporal lobe epilepsy is associated with severely declined dentate neurogenesis in the adult hippocampus. Neurobiol Dis 17: 473-490.

Hattiangady B, Shetty AK (2008). Aging does not alter the number or phenotype of putative stem/progenitor cells in the neurogenic region of the hippocampus. Neurobiol Aging 29: 129-147.

Hattiangady B, Shetty AK (2010). Decreased neuronal differentiation of newly generated cells underlies diminished neurogenesis in chronic temporal lobe epilepsy. Hippocampus 20: 97-112.

Hattiangady B, Shetty AK (2012). Neural stem cell grafting counteracts hippocampal injury-mediated impairments in mood, memory, and neurogenesis. Stem Cells Transl Med 1: 696-708.

Herrero AI, Sandi C, Venero C (2006). Individual differences in anxiety trait are related to spatial learning abilities and hippocampal expression of mineralocorticoid receptors. Neurobiol Learn Mem 86: 150-159.

Hyams KC, Wignall FS, Roswell R (1996). War syndromes and their evaluation: from the US Civil War to the Persian Gulf War. Ann Intern Med 125: 398-405.

Kempermann G, Gast D, Gage FH (2002). Neuroplasticity in old age: sustained fivefold induction of hippocampal neurogenesis by long-term environmental enrichment. Ann Neurol 52: $135-143$. 
Kheirbek MA, Klemenhagen KC, Sahay A, Hen R (2012). Neurogenesis and generalization: a new approach to stratify and treat anxiety disorders. Nat Neurosci 15: 1613-1620.

Kohman RA, Rhodes JS (2013). Neurogenesis, inflammation and behavior. Brain Behav Immun 27: 22-32.

Krishnadas R, Cavanagh J (2012). Depression: an inflammatory illness? Neurol Neurosurg Psychiatry 83: 495-502.

Li L, Shou Y, Borowitz JL, Isom GE (2001). Reactive oxygen species mediate pyridostigmine-induced neuronal apoptosis: involvement of muscarinic and NMDA receptors. Toxicol Appl Pharmacol 177: 17-25.

Li X, Spence JS, Buhner DM, Hart J Jr, Cullum CM, Biggs MM, Hester AL, Odegard TN, Carmack PS, Briggs RW, Haley RW (2011). Hippocampal dysfunction in Gulf war veterans: investigation with ASL perfusion MR imaging and physostigmine challenge. Radiology 261: 218-225.

Malberg JE, Eisch AJ, Nestler EJ, Duman RS (2000). Chronic antidepressant treatment increases neurogenesis in adult rat hippocampus. J Neurosci 20: 9104-9110.

Manganas LN, Zhang X, Li Y, Hazel RD, Smith SD, Wagshul ME et al (2007). Magnetic resonance spectroscopy identifies neural progenitor cells in the live human brain. Science 318: 980-985.

Menon PM, Nasrallah HA, Reeves RR, Ali JA (2004). Hippocampal dysfunction in Gulf War Syndrome. A proton MR spectroscopy study. Brain Res 1009: 189-194.

Meshi D, Drew MR, Saxe M, Ansorge MS, David D, Santarelli L et al (2006). Hippocampal neurogenesis is not required for behavioral effects of environmental enrichment. Nat Neurosci 9: 729-731.

Montaron MF, Drapeau E, Dupret D, Kitchener P, Aurousseau C, Le Moal M et al (2006). Lifelong corticosterone level determines age-related decline in neurogenesis and memory. Neurobiol Aging 27: 645-654.

Narahashi T (1985). Nerve membrane ionic channels as the primary target of pyrethroids. Neurotoxicol 6: 3-22.

Odegard TN, Cooper CM, Farris EA, Arduengo J, Bartlett J, Haley R (2012). Memory impairment exhibited by veterans with Gulf war illness. Neurocase, 23 April 2013 (e-pub ahead of print).

Parihar VK, Hattiangady B, Kuruba R, Shuai B, Shetty AK (2011). Predictable chronic mild stress improves mood, hippocampal neurogenesis and memory. Mol Psychiatry 16: 171-183.

Pereira AC, Huddleston DE, Brickman AM, Sosunov AA, Hen R, McKhann GM et al (2007). An in vivo correlate of exerciseinduced neurogenesis in the adult dentate gyrus. Proc Natl Acad Sci USA 104: 5638-5643.

Perera TD, Dwork AJ, Keegan KA, Thirumangalakudi L, Lipira $\mathrm{CM}$, Joyce $\mathrm{N}$ et al (2011). Necessity of hippocampal neurogenesis for the therapeutic action of antidepressants in adult nonhuman primates. PLoS One 6: e17600.
Ray DE, Fry JR (2006). A reassessment of the neurotoxicity of pyrethroid insecticides. Pharmacol Ther 111: 174-193.

Santarelli L, Saxe M, Gross C, Surget A, Battaglia F, Dulawa S et al (2003). Requirement of hippocampal neurogenesis for the behavioral effects of antidepressants. Science 301: 805-809.

Shetty AK, Hattiangady B, Rao MS (2009). Vulnerability of hippocampal GABA-ergic interneurons to kainate induced excitotoxic injury during old age. J Cell Mol Med 13: 2408-2423.

Shetty AK, Rao MS, Hattiangady B, Zaman V, Shetty GA (2004). Hippocampal neurotrophin levels after injury: Relationship to the age of the hippocampus at the time of injury. J Neurosci Res 78: $520-532$.

Small SA, Schobel SA, Buxton RB, Witter MP, Barnes CA (2011). A pathophysiological framework of hippocampal dysfunction in ageing and disease. Nat Rev Neurosci 12: 585-601.

Snyder JS, Soumier AM, Brewer M, Pickel J, Cameron HA (2011). Adult hippocampal neurogenesis buffers stress responses and depressive behavior. Nature 476: 458-461.

Steele L (2000). Prevalence and patterns of Gulf war illness in Kansas veterans: association of symptoms with characteristics of person, place, time of military service. Am J Epidemiol 152: 992-1002.

Steele L, Sastre A, Gerkovich MM, Cook MR (2012). Complex factors in the etiology of Gulf War illness: wartime exposures and risk factors in veteran subgroups. Environ Health Perspect 120: $112-118$.

Torres-Altoro MI, Mathur BN, Drerup JM, Thomas R, Lovinger DM, O'Callaghan JP et al (2011). Organophosphates dysregulate dopamine signaling, glutamatergic neurotransmission, and induce neuronal injury markers in striatum. J Neurochem 119: 303-313.

Treit D, Fundytus M (1998). Thigmotaxis as a test for anxiolytic activity in rats. Pharmacol Biochem Behav 31: 959-962.

van Praag H, Shubert T, Zhao C, Gage FH (2005). Exercise enhances learning and hippocampal neurogenesis in aged mice. J Neurosci 25: 8680-8685.

Veltri JC, Osimitz TG, Bradford DC, Page BC (1994). Retrospective analysis of calls to poison control centers resulting from exposure to the insect repellent $\mathrm{N}, \mathrm{N}$-diethyl-m-toluamide (DEET) from 1985-1989. J Toxicol Clin Toxicol 32: 1-16.

Vijverberg HP, van den Bercken J (1990). Neurotoxicological effects and the mode of action of pyrethroid insecticides. Crit Rev Toxicol 21: 105-126.

Vythilingam M, Luckenbaugh DA, Lam T, Morgan CA, Lipschitz D, Charney DS et al (2005). Smaller head of the hippocampus in Gulf War-related posttraumatic stress disorder. Psychiatry Res 139: 89-99.

Wang J, Gallagher D, DeVito LM, Cancino GI, Tsui D, He L et al (2012). Metformin activates an atypical PKC-CBP pathway to promote neurogenesis and enhance spatial memory formation. Cell Stem Cell 11: 23-35.

Supplementary Information accompanies the paper on the Neuropsychopharmacology website (http://www.nature.com/npp) 